\title{
Extraordinary radiation super-sensitivity accompanying with sorafenib combination therapy: what lies beneath?
}

\author{
Jayoung Lee, MD, PhD, Ju Hye Lee, MD, Hanbin Yoon, PhD, Ho Jeong Lee, MS, \\ Hosang Jeon, PhD, Jiho Nam, MD \\ Department of Radiation Oncology, Pusan National University Yangsan Hospital, Yangsan, Korea
}

Primary liver tumor, especially hepatocellular carcinoma (HCC), is a common cause of cancer death worldwide. The incidence is generally higher in Asian countries than in western countries. Carcinogenesis of HCC is often associated with hepatitis viral infections. Current standard treatment of HCC is surgical resection or transplantation in patients with early stage disease. However, the patient with advanced stage disease, surgical resection is often limited. Sorafenib or other treatment modalities are not so effective as well. We report a case of unusual radiation super-sensitivity in advanced stage HCC, and review the literature.

Keywords: Hepatocellular carcinoma, Radiotherapy, Sorafenib

\section{Introduction}

Hepatocellular carcinoma $(\mathrm{HCC})$ is the most common type of primary liver malignancy [1]. Treatment of HCC is quite challenging for their poor baseline liver function, and combined medical illness. For early stage tumors, surgical resection is the first choice of curative treatment. However, not so many patients are suitable for surgery. Recently, advanced HCC is often treated with sorafenib, however its treatment results are not satisfactory yet [2].

Old data regarding radiotherapy (RT) to the primary liver tumor was not good because of normal liver toxicity. With advanced RT techniques, increasing number of patients receiving $R T$ for either curative or palliative intent. When the patient has advanced liver disease, the utility of RT is still limited due to their poor treatment response [3].

Here we have reported a case of unusual and unexplained super-sensitivity to combined sorafenib and RT.

\section{Case Report}

A 63-year-old woman was admitted to our cancer center in 2015, complaining of right upper abdominal pain. Otherwise she was healthy with good general condition. From radiologic evaluations in other hospital, she was diagnosed with primary HCC associated with hepatitis B virus and liver cirrhosis. Additional computed tomography (CT) and magnetic resonance (MR) imaging for staging workup showed that over $16 \mathrm{~cm}$ mass at the right hepatic lobe with tumor thrombus in the right portal and main portal vein (Fig. 1). Right hepatic vein and suprahepatic inferior vena cava were also involved. Multiple portocaval and para-aortic lymph nodes were enlarged. At the time of initial visit, her alpha fetoprotein (AFP) level was $>4,340 \mathrm{ng} / \mathrm{mL}$ (normal range [NR], 0.1 to $9.0 \mathrm{ng} / \mathrm{mL}$ ), and the results of liver function tests are aspartate transaminase (AST) $298 \mathrm{IU} / \mathrm{L}(\mathrm{NR}, 0$ to $35 \mathrm{IU} / \mathrm{L})$, alanine transaminase (ALT) $31 \mathrm{IU} / \mathrm{L}(\mathrm{NR}, 0$ to $35 \mathrm{IU} / \mathrm{L})$, alkaline

Received 19 May 2017, Revised 24 May 2017, Accepted 31 May 2017.

Correspondence: Jiho Nam, MD, Department of Radiation Oncology, Pusan National University Yangsan Hospital, 20 Geumoro, Mulgeum-eup, Yangsan 50612, Korea. Tel: +82-55-360-3453, Fax: +82-55-360-3449, E-mail: jihonam@hanmail.net

(c) This is an Open Access article distributed under the terms of the Creative Commons Attribution Non-Commercial License (http://creativecommons.org/ licenses/by-nc/4.0/) which permits unrestricted non-commercial use, distribution, and reproduction in any medium, provided the original work is properly cited.

www.e-roj.org 
phosphatase $299 \mathrm{IU} / \mathrm{L}(\mathrm{NR}, 30$ to $120 \mathrm{IU} / \mathrm{L})$, and total bilirubin $1.1 \mathrm{mg} / \mathrm{dL}$ (NR, 0.3 to $1.2 \mathrm{mg} / \mathrm{dL}$ ), respectively.

She was recommended for the treatment with either trans-arterial chemoembolization (TACE) or sorafenib. She refused TACE for concerning the toxicity. Her initial treatment began with sorafenib for palliative aim. Following CT scan showed that the disease was slightly progressed. Because her abdominal pain was also persisted, sorafenib alone appeared to be not enough to control her disease, then we decided to combine RT to liver expecting additional local control and symptom palliation.

Considering the tumor size and limited normal liver volume, her RT was given with intensity-modulated radiotherapy (IMRT) technique using conventional fractionated schedule. Almost entire liver was included in the planning target volume due to the huge right lobe tumor and tumor thrombus.

During the course of RT, abdominal CT scan was repeated

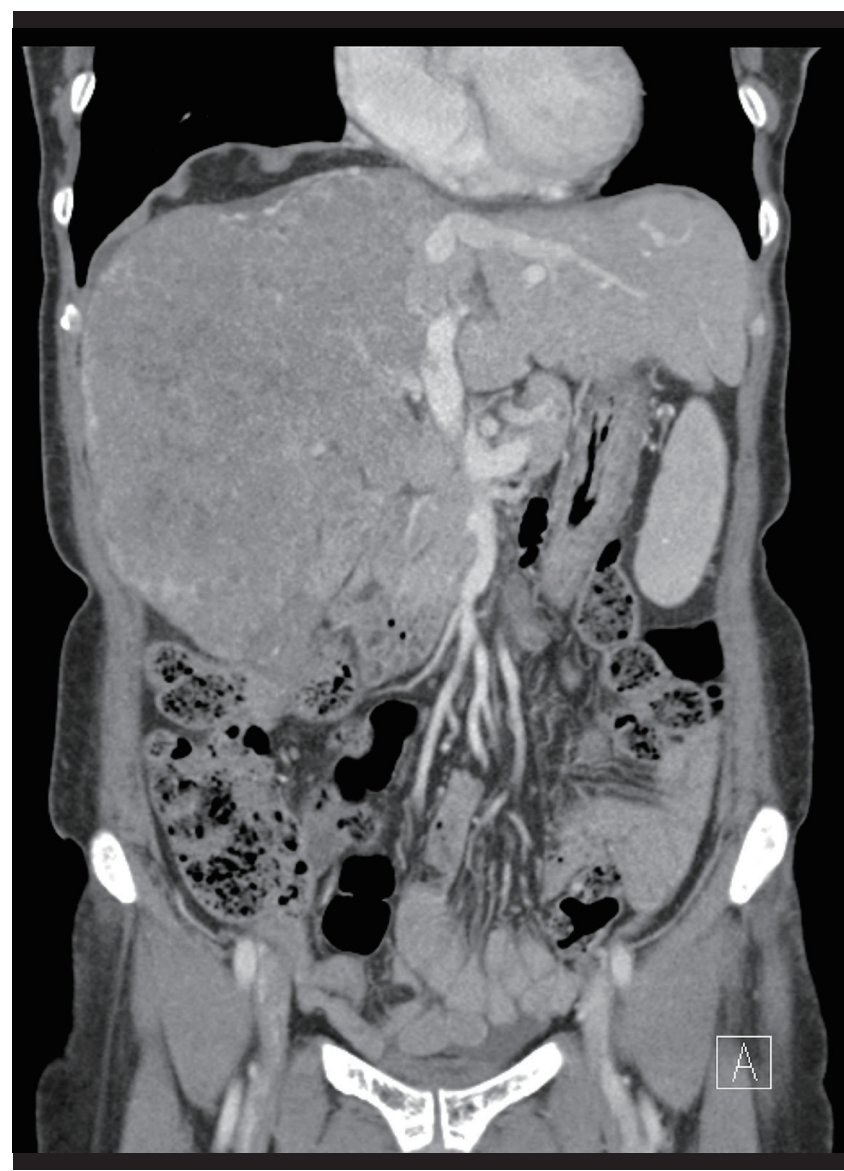

Fig. 1. Huge right lobe hepatocellular carcinoma with portal vein thrombosis was found on pre-treatment computed tomography image. The tumor was occupying almost entire right lobe of the liver. Initially measured tumor volume was around 3,500 mL. for measuring tumor response. Her liver tumor was markedly reduced even after 3 weeks of RT. Because the tumor volume change was so prominent, hepatic tumor rupture was initially suspected rather than the tumor response. However, there were no symptom and clinical and radiologic finding of tumor rupture. Laboratory test results also did not significantly change from the initial test results. Her abdominal pain was even subsided during the course.

Because the tumor volume change was so significant, RT plan modification was necessary to adequately cover tumor mass and to spare surrounding normal organs.

After completion of RT with a total dose of 50 Gy in 5 weeks, serum levels of AFP decreased to $180 \mathrm{ng} / \mathrm{mL}$. Her blood tests for the liver function was also slightly improved compared to the initial values. Sorafenib was given continuously.

Six months later, her abdominal CT scan showed remarkable hepatic tumor regression (Fig. 2).

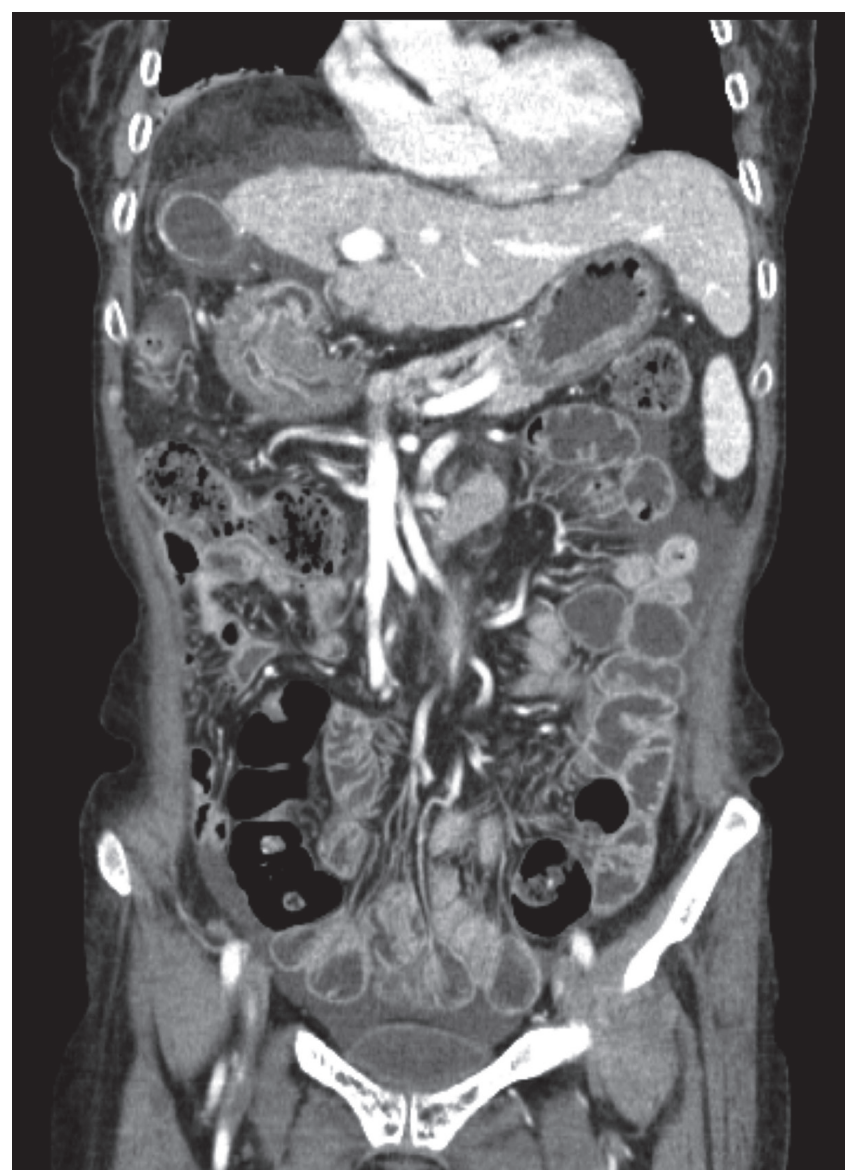

Fig. 2. Liver tumor had markedly reduced on follow-up abdomen computed tomography image. 
However, she had cough and mild dyspnea and upper abdominal distension with slight general weakness. Thoracic CT scan showed radiation pneumonitis (Fig. 3), and gastric ulcer was diagnosed on endoscopic exam. Hospitalization and supportive care was required.

Nine months later, following radiologic studies revealed no progression of liver disease, however, new appearance of peritoneal seeding was found on abdominal CT scan.

She was discharged for hospice care.

\section{Discussion}

Primary liver cancer is one of the main cause of cancer death worldwide. Most of primary liver cancer is HCC. It is reportedly more common in the regions of Asia and Africa. The incidence of HCC is $0.01 \%-0.02 \%$ per year, and gradually increasing in many countries, especially in Europe and America [1].

$\mathrm{HCC}$ are generally associated with various risk factors, i.e., hepatitis viruses, liver cirrhosis, aflatoxin, alcohol consumption, etc. More than two-thirds of virus related HCCs are caused by the hepatitis B virus infection. Nationwide hepatitis B virus vaccination program has greatly contributed in reducing the risk of HCC development. HCC surveillance is also known to be effective for decreasing cancer mortality [4].

Current treatment options for HCC are primarily surgical resection, transplantation, TACE, targeted agents, and so on [2]. Tumor stage and baseline liver function are important factors to select best treatment modality for the patient.

For the early stage HCC, curative resection alone is successful for long-term survival. However, for the patient with advanced stage disease, surgery is not usually feasible. Systemic targeted agents, such as sorafenib, is recommended for the treatment of the advanced disease.

Role of RT for the treatment of liver cancer is unclear. Although there are many published literature regarding the use of RT for the selected patient groups might be effective, the clinical significance has been still debating.

Historically, curative RT for HCC was not feasible for their lower efficacy and severe toxicity. Liver RT has been performed in limited patients group for symptom palliation for liver pain or symptoms associated metastatic disease. However, recent advanced RT techniques may offer promising results. Emerging stereotactic ablative RT technique can safely deliver higher dose to liver tumor with relatively lower surrounding normal organ dose. Local control rates from published reports are approximately $90 \%$ at 2-3 years [3]. Even though the number of patients were small and not randomized prospectively, most

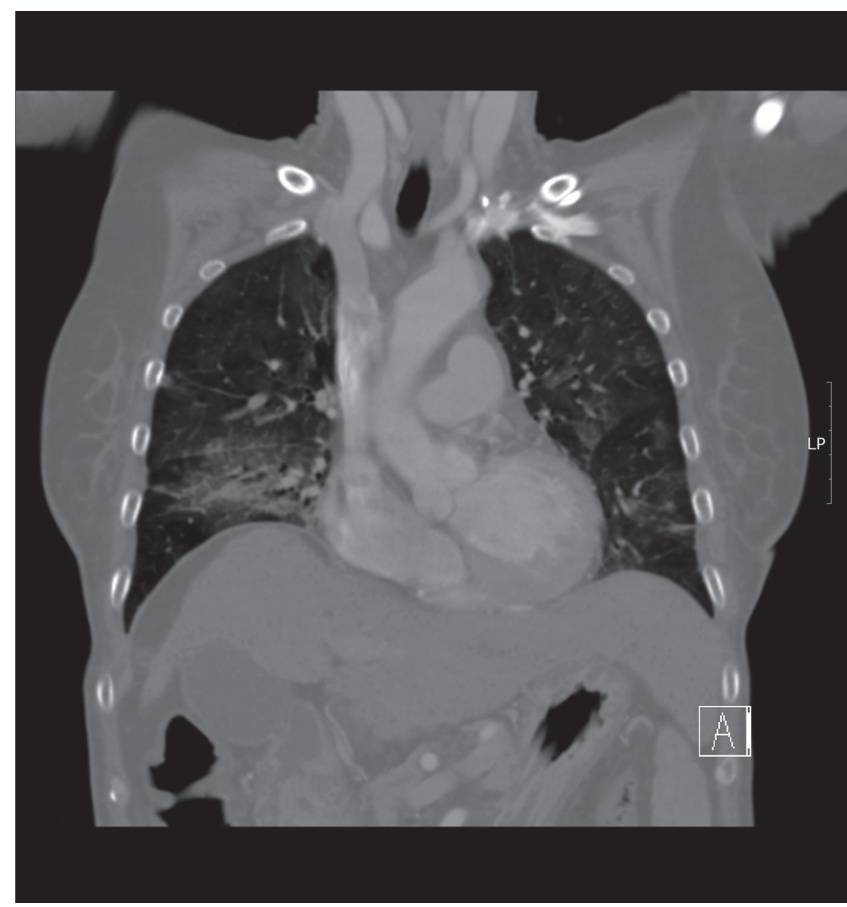

Fig. 3. Follow-up chest computed tomography scan revealed the radiation pneumonitis on the right lower lung.

of the studies consistently reported excellent tumor control rates. It is not easy to combine those results as they have used the various treatment schedules, such as 24 Gy to 60 Gy in 3 to 5 fractions.

There are some studies reported using chemotherapy agents as a radiation sensitizer for the treatment of HCC. Cisplatin was used as a classic radiosensitizer in other cancer for more than 30 years. And doxorubicin and 5-fluorouracil are also available. Their results were somewhat mixed, so additional clinical investigation may be required [3]. Newer molecular targeted agents are not often used as a radiosensitizer yet. In other cancers, sorafenib has been reported promising results when combined with RT.

Several clinical trials testing the efficacy of the combined sorafenib and RT in advanced HCC patients are ongoing $[5,6]$. Treatment responses in the available reports appeared to be somewhat superior, but overcoming treatment toxicities might be challenging.

In our case, the treatment response was unexpectedly prominent comparing with the ordinary responses of each treatment modality. We have tried to reduce the treatment associated toxicities with modern RT techniques, but the tumor volume shrinkage was too fast to catch up with. Dose volume histogram analysis of initial RT plan with adaptive simulation 


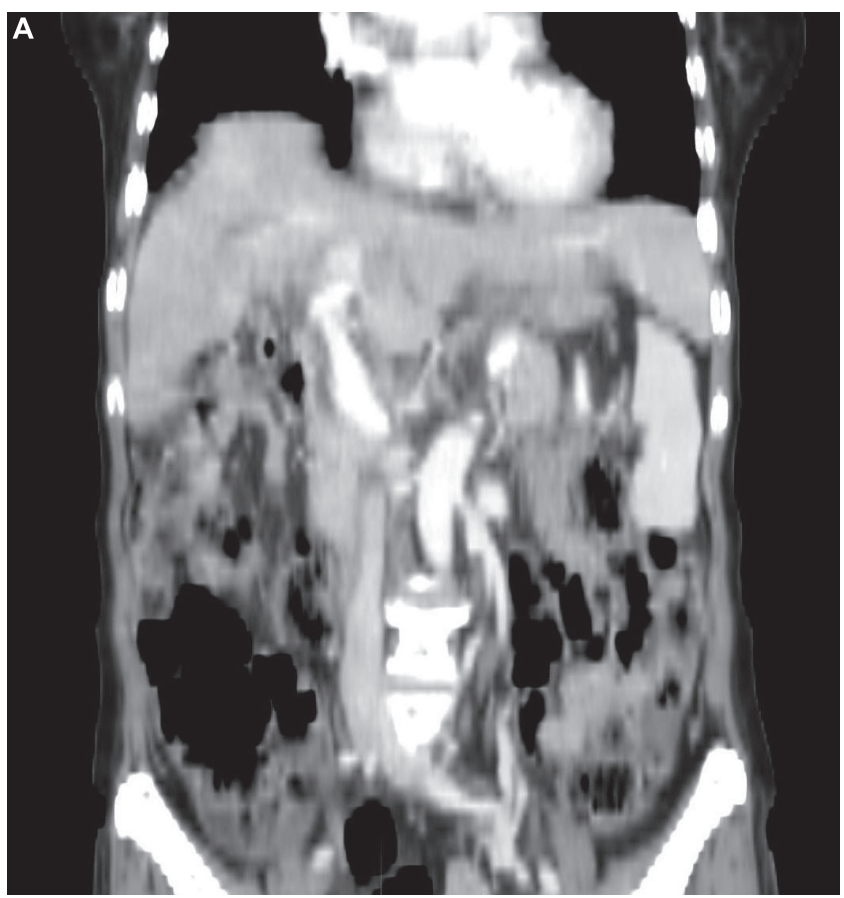

Fig. 4. Patient's re-planning computed tomography (CT) image (A) and the dose volume histogram (DVH) comparison of initial radiotherapy plan $(\mathrm{B})$ with the new adaptive $\mathrm{CT}$ simulation image (C) were presented. There were significant right shifts in DVH curves of normal organs due to the liver tumor volume change. GTV, gross tumor volume.

CT image results are shown in Fig. 4. Current sophisticated $\mathrm{RT}$ techniques do not provide any practical methods for daily tumor volume change adaptation yet. We have changed her RT plan three times during the course to avoid excess radiation dose/volume to normal organs. Nevertheless, she had more severe complications than expected as usual.

In conclusion, treatment of advanced HCCs has been challenging for several decades. Multimodal treatments may be helpful to improve treatment outcomes. Although the role of RT in the treatment of HCC needs to be determined with further clinical trials, like our case, appropriate combination of multimodal treatment options may contribute in successful treatment even in patients with advanced disease.

\section{Conflict of Interest}

No potential conflict of interest relevant to this article was reported.
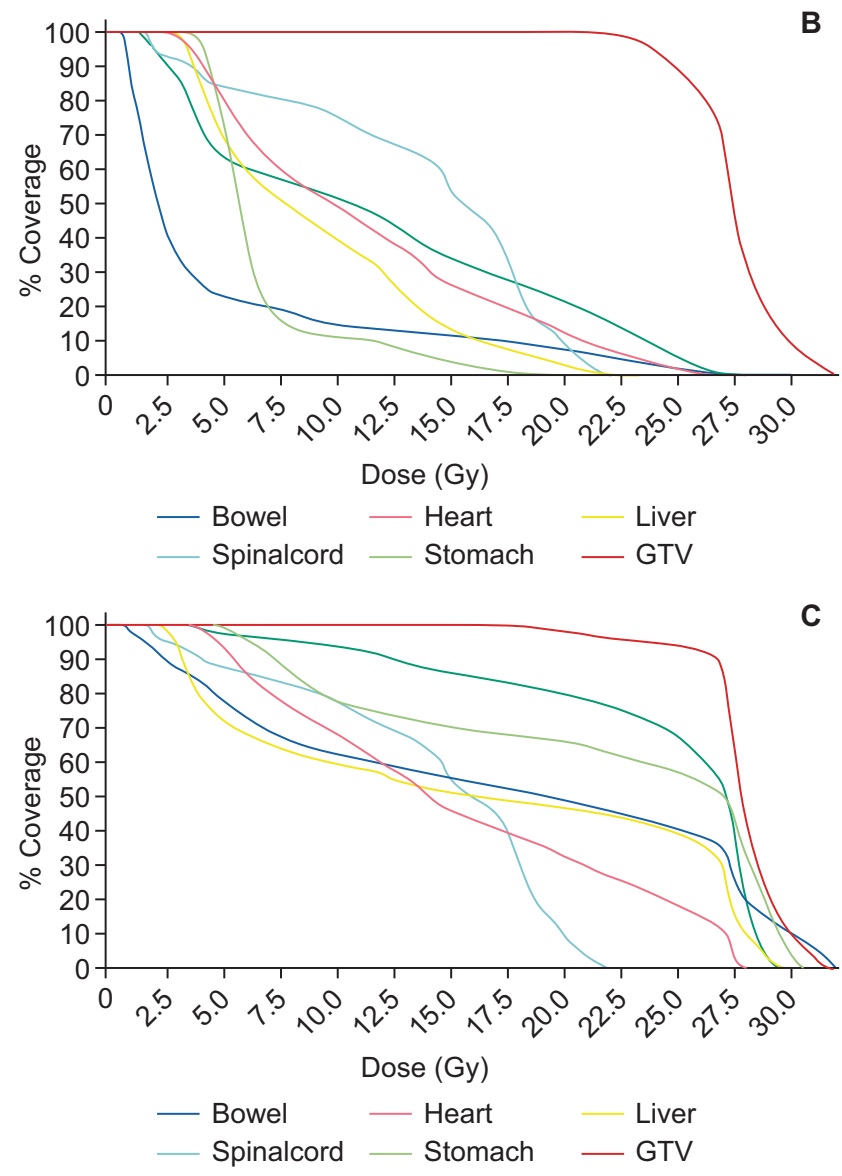

References

1. Mittal S, El-Serag HB. Epidemiology of hepatocellular carcinoma: consider the population. J Clin Gastroenterol 2013;47 Suppl:S2-6.

2. Raza A, Sood GK. Hepatocellular carcinoma review: current treatment, and evidence-based medicine. World J Gastroenterol 2014;20:4115-27.

3. Ohri N, Dawson LA, Krishnan S, et al. Radiotherapy for hepatocellular carcinoma: new indications and directions for future study. J Natl Cancer Inst 2016;108;djw133.

4. McGlynn KA, London WT. The global epidemiology of hepatocellular carcinoma: present and future. Clin Liver Dis 2011;15:223-43, vii-x.

5. Brade AM, Ng S, Brierley J, et al. Phase 1 Trial of sorafenib and stereotactic body radiation therapy for hepatocellular carcinoma. Int J Radiat Oncol Biol Phys 2016;94:580-7.

6. Chen SW, Lin LC, Kuo YC, Liang JA, Kuo CC, Chiou JF. Phase 2 study of combined sorafenib and radiation therapy in patients with advanced hepatocellular carcinoma. Int J Radiat Oncol Biol Phys 2014;88:1041-7. 